Louisiana State University

LSU Digital Commons

Faculty Publications

Department of Biological Sciences

$1-1-2012$

\title{
Contextual chemosensory urine signaling in an African cichlid fish
}

\author{
Karen P. Maruska \\ Stanford University \\ Russell D. Fernald \\ Stanford University
}

Follow this and additional works at: https://digitalcommons.Isu.edu/biosci_pubs

\section{Recommended Citation}

Maruska, K., \& Fernald, R. (2012). Contextual chemosensory urine signaling in an African cichlid fish. Journal of Experimental Biology, 215 (1), 68-74. https://doi.org/10.1242/jeb.062794

This Article is brought to you for free and open access by the Department of Biological Sciences at LSU Digital Commons. It has been accepted for inclusion in Faculty Publications by an authorized administrator of LSU Digital Commons. For more information, please contact ir@lsu.edu. 


\title{
RESEARCH ARTICLE \\ Contextual chemosensory urine signaling in an African cichlid fish
}

\author{
Karen P. Maruska* and Russell D. Fernald \\ Department of Biology, Stanford University, 371 Serra Mall, Stanford, CA 94305, USA \\ *Author for correspondence (maruska@stanford.edu)
}

Accepted 4 October 2011

\begin{abstract}
SUMMARY
Chemosensory signaling is crucial for communication in many fish species, but little is known about how signalers modulate chemical output in response to sensory information and social context. Here, we tested the hypothesis that dominant male African cichlid fish (Astatotilapia burtoni) use urine signals during social interactions, and demonstrate that this signaling depends on social context (reproductive; territorial) and on available sensory information (visual cues; full interaction). We injected males with dye to visualize urine pulses and exposed them to full sensory information or visual cues alone of four types: (1) dominant male; (2) gravid (reproductively receptive) females; (3) mouth-brooding (non-receptive) females; or (4) control (no fish). We found that males released urine sooner and increased their urination frequency when visually exposed to gravid females as compared with mouth-brooding females and or no-fish controls. While males could distinguish female reproductive states using visual cues alone, courtship behavior rates were $\sim 10$-fold higher when they fully interacted with gravid females compared with receiving visual cues alone. Males also increased their urination and territorial behaviors when exposed to another male, suggesting that chemical signals may convey information on dominance status. These data support the hypothesis that dominant males use urine as a chemical signal and adjust the frequency of their urine output based on contextual information.
\end{abstract}

Key words: Astatotilapia burtoni, communication, olfactory, reproduction, teleost, urine.

\section{INTRODUCTION}

Multimodal signals that may include combinations of visual, acoustic, mechanosensory, olfactory and tactile information are typically used during both intra- and inter-sexual social interactions across taxa (Partan and Marler, 1999; Candolin, 2003; Narins et al., 2005; Van Staaden and Smith, 2011). Animals use these signals to convey information about their sex, breeding condition, social status and quality as a prospective mate, which have significant consequences for survival and fitness. Although integration of multiple sensory signals is crucial for an animals' ability to respond appropriately during social interactions, little is known about the role of individual signal components in a complex display, or how information from one sensory modality may modulate the signaling output of another. For example, is merely seeing another conspecific sufficient to influence contextual chemical signaling, and are specific sensory cues necessary to elicit a particular appropriate behavioral response? While many studies examine both unimodal and multimodal communication from the receiver's perspective (Partan and Marler, 1999; Candolin, 2003), comparatively little is known about how reception of contextual and sensory information then feeds back to influence the signaler, especially in fish. Thus, to fully understand communicative signal function and evolution, it is also important to examine the contextual cues that might modulate the behavior and signal output of the sender.

Cichlid fishes are excellent models to test hypotheses about the mechanisms and importance of multimodal communication from the signalers' perspective because they are known to use multiple sensory cues to coordinate their complex social behaviors. In particular, cichlid species are incredibly diverse in their reproductive, social and parental care strategies, which have produced numerous adaptations in their communication systems (Van Staaden and Smith, 2011). Astatotilapia burtoni is a maternal mouth-brooding cichlid species endemic to shallow shore pools of Lake Tanganyika, Africa. Dominant males ( $\sim 10-30 \%$ of the population) are brightly colored and aggressively defend territories in a lek-like social system where they court and spawn with females, while subordinate males are reproductively suppressed, do not defend territories, and school with and are similar in coloration to females (Fernald and Hirata, 1977). Receptive females will enter the spawning territory area, choose one or more males to spawn with, and then care for the developing embryos in their mouths for $\sim 2$ weeks until releasing them as free-swimming fry. Previous studies demonstrated that $A$. burtoni males could discriminate between gravid (reproductively receptive) and unreceptive females based on smell alone (Crapon de Caprona, 1980), and that their olfactory system was sensitive to conjugated steroids (Robison et al., 1998; Cole and Stacey, 2006), suggesting that females may release compounds that are used by males to distinguish reproductive readiness. However, while we know that both visual and acoustic signals are used by dominant males during courtship (Fernald, 1977; Fernald and Hirata, 1977; Maruska and Fernald, 2010a), it is not yet known whether they also emit chemical signals during reproductive interactions with conspecifics.

Visual signaling, in the form of both body coloration and stereotypical behavioral displays, is extremely important in both territorial and reproductive contexts in many fish species, but whilst visual signals can function over long distances, in close quarters chemical signals may also be important. Chemosensory signaling is a key communication channel in fishes and has been best studied in the goldfish Carrasius auratus where both males and females release pheromones to prime the opposite sex and synchronize spawning (Dulka et al., 1987; Sorensen, 1992; Appelt and Sorensen, 1999; Stacey and Sorensen, 2005; Appelt and Sorensen, 2007; 
Stacey, 2011). Many other fish species also use pheromones to influence the behavior and physiology of conspecifics, which has important consequences for reproductive success (Almeida et al., 2005; Gerlach, 2006; Barata et al., 2008b; Barata et al., 2008a; Rosenthal et al., 2011), and this phenomenon is conserved through mammals (Dulac and Torello, 2003; Swaney and Keverne, 2009). One mode of pheromone release in fishes is via urine pulses; however, few studies have examined how individuals might alter their urine release for use as a contextual social signal. For example, male swordtails (Xiphophorus spp.) urinate more frequently in the presence and proximity of conspecific females (Rosenthal et al., 2011), male tilapia Oreochromis mossambicus increase their urination frequency when exposed to receptive but not unreceptive females (Almeida et al., 2005; Barata et al., 2007; Barata et al., 2008b), and levels of urinary conjugated steroids (e.g. 11ketotestosterone, 11-KT) are higher following social interactions in several cichlid fish species (Oliveira et al., 1996; Hirschenhauser et al., 2004; Hirschenhauser et al., 2008). However, previous studies have not specifically addressed which sensory cues are used by the chemical signaler to adjust their signal as a function of the sex, reproductive condition or social status of the recipient.

In territorial species that establish dominance hierarchies, dominant males may also use chemical signals, along with visual signals and aggressive behaviors, to broadcast their social status to rival males. For example, male $O$. mossambicus actively signal their dominance status via urinary odorants that may modulate aggression levels in conspecific males (Barata et al., 2007), but as stated above, little is known about which sensory cues are salient for initiation of the investment in chemical signaling. Dominant $A$. burtoni males use visual signals such as eye-bar darkening, intensification of body coloration and aggressive behavioral displays to visually and physically suppress subordinate males, thus preventing them from holding territories and spawning with females (Fernald and Hirata, 1977; Fernald, 2009). It is not known, however, whether dominant males also use chemical signals as an additional cue to convey status and suppress the reproductive behavior and physiology of subordinates.

Here, we first asked whether dominant male $A$. burtoni use urine pulses as chemical social signals, and then tested the hypothesis that this signaling depends both on social context (reproductive and territorial) and on the type of available sensory information (full sensory interaction $v s$ only visual cues). We show for the first time that dominant male $A$. burtoni use urine pulses as contextual social signals for both reproductive and territorial interactions, and that visual cues alone can increase male chemical signaling while full contact with all available sensory information is required to elicit more stereotypical courtship and dominance behaviors.

\section{MATERIALS AND METHODS Animals}

Adult cichlid fish $A$. burtoni (Gunther 1893) were derived from wildcaught stock in Lake Tanganyika, Africa, maintained in aquaria under environmental conditions that mimic their natural habitat $\left(28^{\circ} \mathrm{C} ; \mathrm{pH} 8.0 ; 12 \mathrm{~h}\right.$ light: $12 \mathrm{~h}$ dark, full spectrum illumination) and fed cichlid flakes (AquaDine, Healdsburg, CA, USA) each morning. These fish have been naturally bred in the laboratory since the original field collection in the late 1970s and exhibit identical territorial and reproductive behaviors to those described in wild populations (Fernald and Hirata, 1977). Aquaria contained gravelcovered bottoms with half terracotta pots that served as shelters and spawning territories. All experimental procedures were approved by the Stanford Administrative Panel for Laboratory Animal Care.

\section{Experimental protocol}

Experiments were conducted in 401 aquaria that were partitioned into three equal-sized compartments $(26 \times 16.5 \times 33 \mathrm{~cm})$ with clear acrylic barriers (Fig. 1A). Clear barriers were permanently sealed to the walls of the aquaria and verified to not permit spreading of olfactory cues to adjacent compartments by showing that colored dye added separately to the water in each compartment did not transfer to adjacent compartments. To eliminate cross-experiment contamination, the experimental tank was also completely drained and cleaned between all trials. A subject dominant male [standard length (SL): $53.9 \pm 3.1 \mathrm{~mm}$ (mean \pm s.d.); body mass $\left(M_{\mathrm{b}}\right)$ : $4.5 \pm 0.8 \mathrm{~g}$; $N=70]$ was placed into the center compartment that contained a single terracotta pot to serve as a territory and shelter, and allowed to acclimate for 2 days while visually exposed to the left compartment that contained a smaller dominant male and two mouth-brooding females ('acclimation fish'). The right compartment was visually blocked by a removable opaque barrier during the acclimation period. In pilot experiments, this acclimation set-up was shown to be necessary to ensure that the subject male established
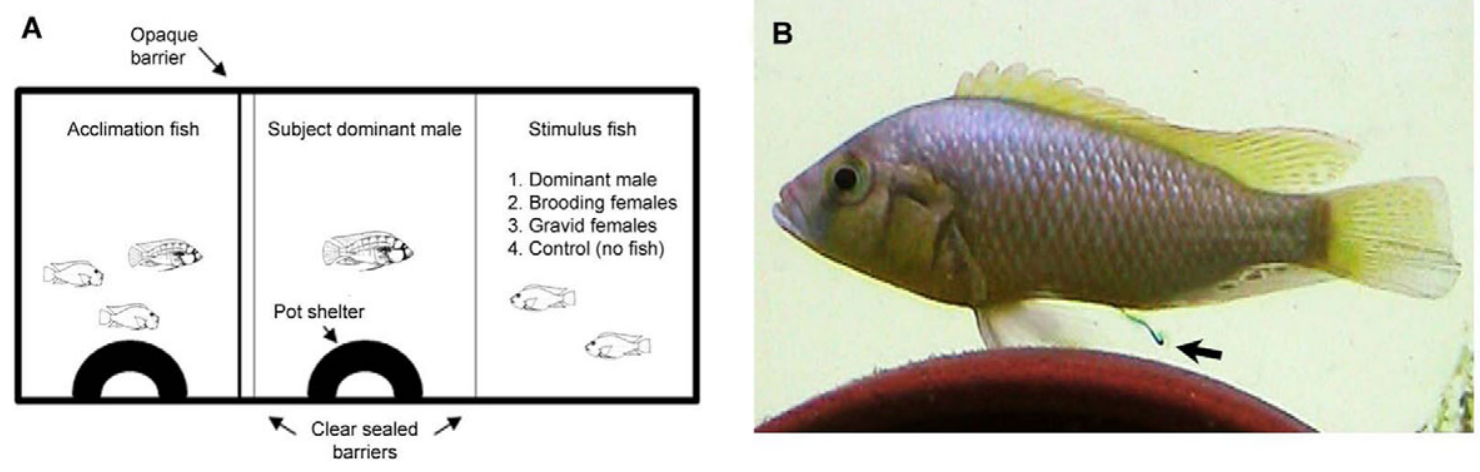

Fig. 1. Experimental protocol and example of pulsatile urine release from a dominant male Astatotilapia burtoni. (A) Experimental tank set-up during stimulus trials. On the day of the experiment, the dye-injected subject dominant male in the center compartment was isolated from the acclimation fish (left) with an opaque barrier (pre-stimulus isolation) and then exposed to one of four different stimulus conditions (right). The subject male was also exposed to the stimulus fish in two different sensory protocols: (1) the opaque barrier separating the subject and stimulus fish was removed to provide only visual cues to the subject, while keeping the fish separated by an impermeable transparent barrier, or (2) the stimulus fish were introduced into the subject males' compartment, providing full sensory information and physical interaction. (B) Example of a urine pulse (arrow) released from a dominant male that was visually exposed to another dominant male. 
A

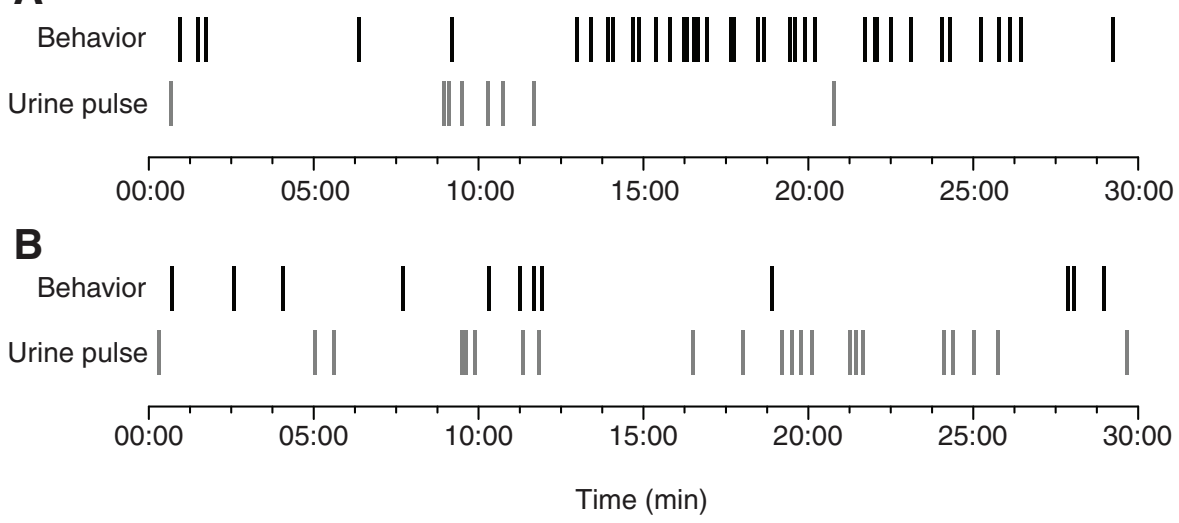

Fig. 2. Urine pulses from dominant male $A$. burtoni were not coincident with courtship or territorial behavioral displays. Examples of the temporal sequence of urine pulses and reproductive or territorial behaviors produced by individual dominant males when exposed to gravid females ( $A$, full sensory interaction) or another dominant male ( $B$, visual cues only). Each vertical mark represents a single urine pulse or behavioral display across the $30 \mathrm{~min}$ trial. Quantified behaviors were either reproductive courtship displays $(A)$ or territorial displays $(B)$. a territory and performed stereotypical dominance behaviors in the experimental tank prior to testing.

\section{Dye injections and experimental procedure}

On the evening before each experiment, the subject male was injected in the left dorsal musculature with $15 \mu \mathrm{l}$ of Patent Blue Violet dye $\left(50 \mathrm{mg} \mathrm{ml}^{-1}\right.$ in $0.9 \%$ sodium chloride; Sigma-Aldrich Inc., St Louis, MO, USA) and placed into a bucket for 5 min to allow any excess dye to leach off the skin. Patent Blue is an innocuous dye that concentrates in the urine and can be readily visualized as urine pulses are expelled from the urogenital opening (Appelt and Sorensen, 2007; Barata et al., 2007) (Fig. 1B). An opaque barrier was put in the tank to shield the acclimation fish in the left compartment and then the subject male was placed back into the center compartment where he was isolated overnight.

On the following morning (08:00-09:00 h), the fish were fed and the experiment began $\sim 10$ min later. All experiments were viewed live by an observer and filmed for later analysis. To obtain baseline urination rates for each individual, the subject male was first observed and filmed in isolation for $30 \mathrm{~min}$ (pre-stimulus). In the first experiment, we tested what role full contact (i.e. all sensory information) had on male urination rates and behavior. This mimics the natural situation where subject males are exposed to multimodal sensory information during reproductive and territorial interactions. After the pre-stimulus isolation period, different stimulus fish that represented either reproductive (female exposure) or territorial (male exposure) contexts were introduced directly into the subject male's compartment: (1) a dominant male ( $N=10)$; (2) two mouth-brooding females $(N=10)$; (3) two gravid (ready-to-spawn) females $(N=10)$; or (4) no fish control $(N=10)$. Each subject male was tested in only one stimulus condition such that 40 different subject males were used for this experiment. All stimulus fish were appropriately size matched to the subject male (brooding females, $\mathrm{SL}=46.80 \pm 3.73 \mathrm{~mm}$, $M_{\mathrm{b}}=2.35 \pm 0.44 \mathrm{~g}$; gravid females, $\mathrm{SL}=48.5 \pm 4.9 \mathrm{~mm}, M_{\mathrm{b}}=3.4 \pm 0.8 \mathrm{~g}$; dominant males, $\mathrm{SL}=52.8 \pm 1.9 \mathrm{~mm}, M_{\mathrm{b}}=4.1 \pm 0.5 \mathrm{~g}$ ), and different unfamiliar stimulus fish were used for each individual trial. Brooding females all had mouths full of pigmented developing embryos and were in the later stages of the $\sim 2$ week maternal care stage. Gravid females all had visibly swollen abdomens prior to daily feeding, which is a proxy for high gonadosomatic index (GSI) and receptivity ( $\sim 97 \%$ of gravid females selected this way have GSI $>4.0$ and generally spawn within a few days; K.P.M. and R.D.F., unpublished observations). Fish were observed and filmed (Canon FS20) during both the pre-stimulus period and the $30 \mathrm{~min}$ stimulus trial. During the stimulus trial, the left opaque barrier remained in place to block visual exposure to the acclimation fish, while the right opaque barrier was removed simultaneously with the introduction of stimulus fish into the subject male's compartment to reveal an empty compartment. Removal of the right barrier was performed in these trials to allow for direct comparisons to the visual cues alone trials described below, where the barrier was also removed. The number of urine pulses and the time of each pulse were quantified, and data were expressed as the percentage change in urination rate (pulses $\mathrm{min}^{-1}$ ) between the pre-stimulus isolation period and the stimulus period for each individual. From the video recordings, the total number of reproductive behaviors (courtship quivers and leading) were quantified when males were exposed to females (gravid and brooding), while territorial behaviors (frontal threats and lateral displays) were quantified during male--male conditions.

In a second experiment, we tested the hypothesis that visual social cues alone could influence dominant male urination rates and dominance behaviors. The experimental protocol was identical to that described above except that removal of the right opaque barrier revealed one of the four different visual stimuli (i.e. a dominant male, two gravid females, two mouth-brooding females or an empty compartment; $N=10$ different subject males per stimulus fish condition). The no-fish control trials were only performed once, as described above for the full interaction experiment, and were also used here for comparisons. Observations and data were collected as above. Comparisons among stimulus fish conditions were made with one-way ANOVA (females) followed by post hoc Student-Newman-Keuls (SNK) tests for pair-wise comparisons, or Student's $t$-tests (males), and comparisons between sensory conditions (visual only or full interaction) were made with Student's $t$-tests (SigmaPlot 11.0, Systat Inc., San Jose, CA, USA).

\section{RESULTS}

Urine pulses were observed from all subject males as blue plumes released from the urogenital opening that were distinct from feces (Fig. 1B). Surprisingly, the majority of urine pulses were not released during any specific behavioral displays but, rather, occurred between behaviors during periods of pause or slow swimming (Fig. 2). Urine release from males in isolation during the pre-stimulus period ranged from 0.028 to 0.083 pulses $\mathrm{min}^{-1}$, while mean ( \pm s.e. $)$ rates during the stimulus period were: control $0.047 \pm 0.013$ pulses $\mathrm{min}^{-1}$; brooding females visual only $0.117 \pm 0.034$ pulses $\mathrm{min}^{-1}$, full interaction $0.167 \pm 0.042$ pulses $\mathrm{min}^{-1}$; gravid females visual only $0.330 \pm 0.052$ pulses $\mathrm{min}^{-1}$, full interaction $0.297 \pm 0.094$ pulses $\mathrm{min}^{-1}$; and dominant male visual only $0.380 \pm 0.073$ pulses $\mathrm{min}^{-1}$, full interaction $0.230 \pm 0.039$ pulses $\mathrm{min}^{-1}$. 


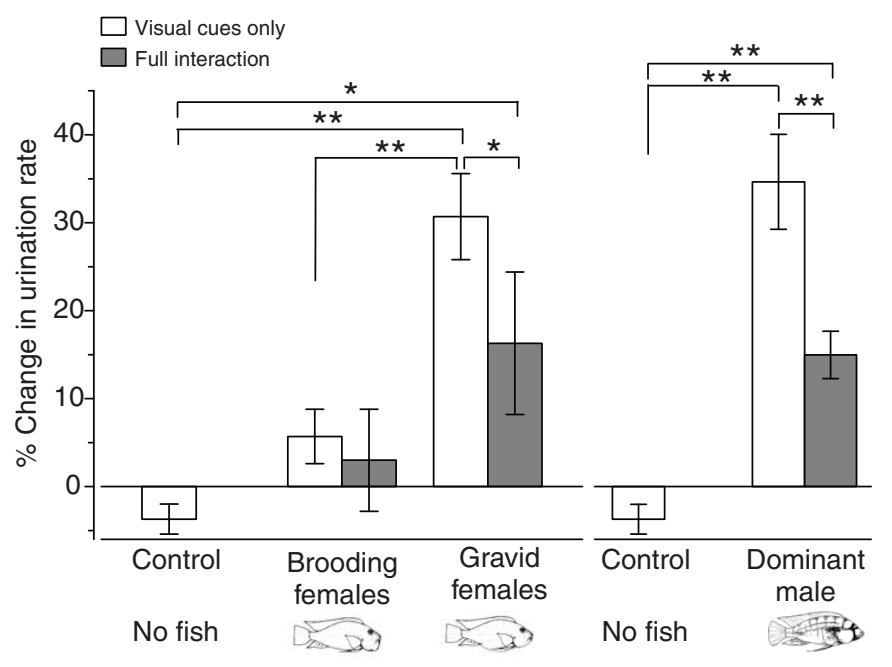

Fig. 3. Dominant male $A$. burtoni alter their chemical urine signaling in response to contextual sensory social cues. The percentage change in urination rate (pulses $\mathrm{min}^{-1}$ between pre-stimulus isolation and stimulus condition) was higher when dominant males were exposed to either another dominant male or to gravid females than when they were exposed to mouth-brooding females or an empty compartment (no-fish control). Urination rates were also higher during the visual only compared with full interaction trials when males were exposed to either gravid females or a dominant male. Lines and asterisks indicate statistical differences: ${ }^{*} P<0.05$; ${ }^{\star \star} P<0.01$. $N=10$ different subject males for each condition. Control bars are the same data set plotted next to both female and male exposure conditions for comparison.

\section{Reproductive context: exposure to females of different reproductive states}

To test whether dominant males would modulate their urine release based on exposure to females of different reproductive states, we allowed males to either fully or only visually interact with gravid (receptive) or brooding (unreceptive) females. The percentage change in urine pulse rate between pre-stimulus and stimulus periods was $\sim 5-6$ times higher when subject males were visually exposed to gravid females than to either brooding females or a control empty compartment (ANOVA, $F_{2,27}=25.94, P<0.001$; SNK, $P<0.001$ ) (Fig.3). In the full interaction trials, the percentage change in urination rate for the gravid female treatment was also higher than for the control (ANOVA, $F_{2,27}=3.62, P=0.040$; SNK, $P=0.049$ ), but it did not differ from the brooding female treatment values $(P>0.05)$. Urination rates were also higher when males were only visually exposed to gravid females compared with the full interaction condition (Student's $t$-test, d.f. $=18, t=-1.48, P=0.037$ ), but rates did not differ between sensory conditions within the brooding female treatment $(P=0.694)$ (Fig. 3).

The latency to the first urine pulse was $\sim 5$ times shorter when subject males were visually exposed to gravid females than when exposed to either brooding females or an empty compartment (ANOVA, $F_{2,27}=7.76, P=0.003$; SNK, $P<0.01$ ) (Fig. 4). In the full interaction trials, the latency to the first urine pulse was also shorter when males were exposed to either gravid females (ANOVA, $F_{2,27}=6.57, P=0.006$; SNK, $\left.P=0.005\right)$ or brooding females $(P=0.011)$ relative to controls, but latency did not differ between gravid and brooding female exposure $(P=0.480)$. Latency did not differ between visual only and full interaction trials for gravid female exposure ( $t$ test, d.f. $=18, t=-0.703, P=0.490)$, but when exposed to brooding females, latency was shorter in full interaction than in visual only trials $(P=0.008)$ (Fig. 4).

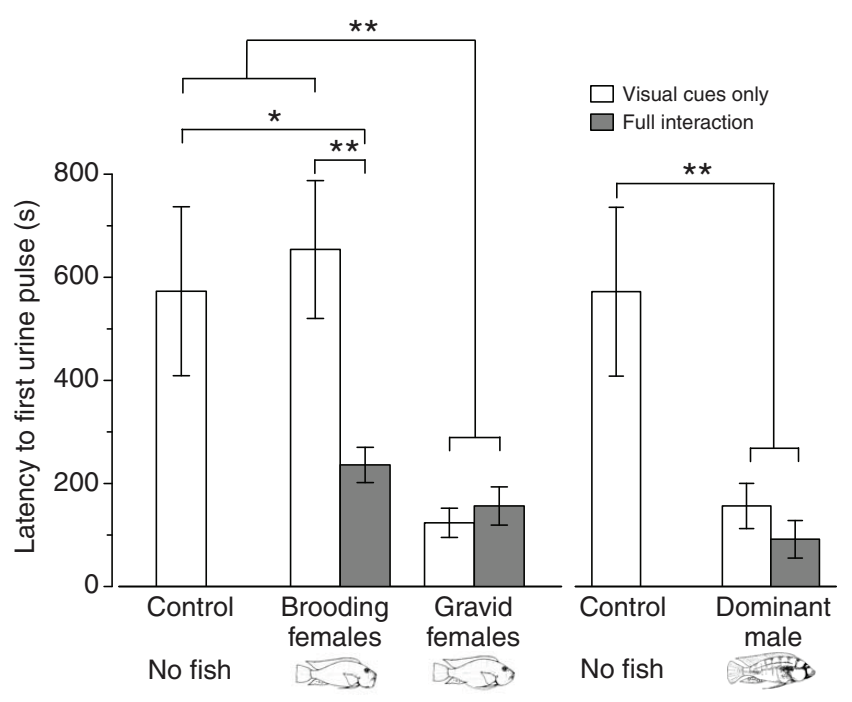

Fig. 4. Dominant male $A$. burtoni change their latency to start chemical urine signaling in response to contextual sensory social cues. Males released their first urine pulse sooner (reduced latency) when exposed to either another dominant male or gravid females, but there was no difference between visual only and full interaction trials for either stimulus. Males also showed a shorter latency when fully exposed to brooding females compared with visual only trials. Lines and asterisks indicate statistical differences: ${ }^{\star} P<0.05$; ${ }^{\star *} P<0.01 . N=10$ different subject males for each condition. Control bars are the same data set plotted next to both female and male exposure conditions for comparison.

The number of reproductive behaviors (courtship quivers and leading) performed by the subject male was significantly higher when he was exposed to gravid females than to both brooding females and control conditions in both visual only (ANOVA, $F_{2,27}=4.43, P=0.022$; SNK, $P<0.05$ ) and full interaction conditions (ANOVA, $F_{2,27}=7.59, P=0.002$; SNK, $P<0.01$ ) (Fig. 5). Rates of these behaviors, however, were $\sim 10$-fold higher when subject males were in full contact with gravid females than with visual exposure alone ( $t$-test, d.f. $=18, t=-2.75, P=0.013$ ), while there was no difference between sensory conditions when exposed to brooding females $(P=0.116)$ (Fig. 5). In the gravid female full interaction trials, increased behavior rates were also often coincident with qualitative intensification of body coloration in subject males (7/10 trials), a phenomenon that did not occur in any of the gravid visual only trials, brooding female trials, or controls ( $0 / 10$ trials for each).

\section{Territorial context: exposure to another dominant male}

To test whether dominant males would also modulate their urine release in territorial contexts, we allowed dominant males to either fully or only visually interact with another size-matched dominant male. Urination rates were significantly higher when subject dominant males were exposed to another dominant male in both visual only ( $t$-test, d.f. $=18, t=-6.88, P<0.001)$ and full interaction trials $(t=-6.29, P<0.001)$ compared with controls (Fig. 3). However, urination rates were also $\sim 2$-fold higher in visual only compared with full interaction trials ( $t$-test, d.f. $=18, t=3.23$, $P=0.005$ ) (Fig. 3).

The latency to the first urine pulse was significantly shorter when males were exposed to another dominant male in both visual only $(t$-test, d.f. $=18, t=2.74, P=0.016)$ and full interaction trials $(t=3.23$, $P=0.006)$ compared with controls, but latencies did not differ between the two sensory conditions ( $t=1.14, P=0.273$ ) (Fig. 4). 


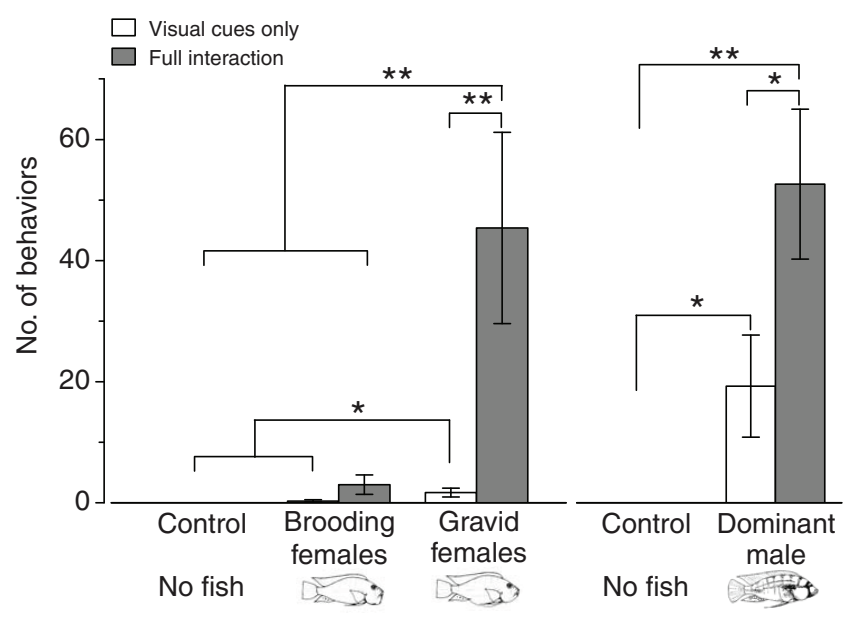

Fig. 5. Dominant male $A$. burtoni alter their rate of courtship and territorial behaviors in response to contextual sensory social cues. The total number of behaviors (mean \pm s.e. per 30 min stimulus trial) was highest when dominant males were fully exposed to either gravid females or another dominant male compared with brooding females or control conditions. Male A. burtoni increased their behaviors when only visually exposed to both gravid females and another dominant male, but rates of these behaviors were $~ 3-$ to 10-fold higher in the full sensory interaction trials. Behaviors quantified for female exposure conditions were courtship quivers and leads, while behaviors for male exposure were lateral displays and frontal threats. Lines and asterisks indicate statistical differences: ${ }^{\star} P<0.05 ;{ }^{* \star} P<0.01 . N=10$ different stimulus males for each condition. Control bars are the same data set plotted next to both female and male exposure conditions for comparison.

Subject males also performed more territorial behaviors (frontal threats and lateral displays) when exposed to another dominant male in both visual only ( $t$-test, d.f. $=18, t=-2.29, P=0.035)$ and full interaction trials ( $t$-test, d.f. $=18, t=-4.23, P<0.001$ ) compared with controls (Fig. 5). The rates of these behaviors were also higher when subject males were in full contact with another dominant male compared with visual exposure alone ( $t$-test, d.f. $=18, t=-2.22$, $P=0.040)$ (Fig. 5).

\section{DISCUSSION}

Our results are the first to demonstrate that dominant male $A$. burtoni modulate their rate of urine release in both reproductive and territorial contexts, suggesting that they use urine as a chemical social signal. Further, we show that rates of reproductive and territorial behavior are significantly lower when males are only visually exposed to other fish, suggesting that information from other sensory modalities such as olfaction is important to elicit stereotypical behaviors. These data support the hypothesis that contextual chemosensory information is important during social interactions in this species, and suggests that $A$. burtoni utilizes a chemical communication system that has the potential to influence both male-male and male-female interactions. Future experiments are needed, however, to directly test how this urine release might influence the behavior of both females and other males. To our knowledge, this is the first study to examine male chemical signaling in both different social contexts (reproductive and territorial) and different sensory conditions (visual only and full sensory) in a single fish species, and highlights the importance of studying multimodal communication in social vertebrates.

Freshwater organisms are hyperosmotic to their environments and must continuously rid their bodies of excess water, and, as a result, urinate frequently. Capitalizing on this physiological constraint, many freshwater fishes have evolved pheromonal signals as a means of communicating important information to potential mates and rivals (Appelt and Sorensen, 1999; Kodric-Brown and Strecker, 2001; Stacey, 2011). Pheromones are typically defined as substances, or mixtures of substances, that are released by individuals into the environment where they induce specific, adaptive and largely innate biological responses in conspecifics that are mutually beneficial to both the sender and receiver (Karlson and Luscher, 1959; Sorensen et al., 2000). The olfactory organs of aquatic animals are constantly exposed to soluble conspecific odorants, and fishes may be predisposed to evolve pheromonal functions for released sex hormones (and other metabolites) because they should provide reliable indicators of important reproductive events and internal physiological state, including nutritional status (Doving, 1976; Fisher and Rosenthal, 2006; Giaquinto et al., 2010). Several freshwater fishes are also known to alter when and where they release urinary pheromones, which is consistent with the hypothesis of a true communication system (benefits both signaler and receiver) designed to optimize detection by the intended receiver (Bradbury and Vehrencamp, 1998; Stacey and Sorensen, 2005; Appelt and Sorensen, 2007; Rosenthal et al., 2011). Here, we have demonstrated that dominant male $A$. burtoni use urine pulses in the context of both mating and territorial dominance presumably to deliver information to conspecifics. The type of information these urine pulses convey, and how this might be used by intended and possibly unintended receivers, however, requires further study.

Male $A$. burtoni had higher urination rates when visually exposed to gravid females relative to brooding females, suggesting that they can distinguish female reproductive state based on visual cues alone. In another study using the Nile tilapia Oreochromis niloticus, female visual cues alone were also sufficient to increase courtship behaviors and GSI in males (Castro et al., 2009). In our study, rates of courtship behavior by $A$. burtoni males given visual cues alone, however, were $\sim 10$-fold lower than when gravid females were in the same compartment and males had access to full sensory information. Previous studies also showed increased courtship behavior in male A. burtoni exposed to water that had housed gravid females (Crapon de Caprona, 1974; Crapon de Caprona, 1980). Thus, it is possible that olfactory information from gravid females is required to prime the male's reproductive system before he invests in potentially energetically demanding visual, and possibly acoustic, courtship displays. In other fishes, for example, exposure to compounds released by receptive females can increase both male reproductive behaviors and activity of the hypothalamic-pituitary-gonadal (HPG) axis (e.g. increased plasma luteinizing hormone levels, milt volume, sperm motility) (for a review, see Stacey, 2011), a phenomenon that also occurs in other taxa including mammals (Coquelin and Bronson, 1979; Johnston and Bronson, 1982; Swaney and Keverne, 2009). The idea that pheromones released by the female can have both physiological 'primer' and behavioral 'releaser' effects on males is further supported by the fact that male $A$. burtoni urination rates were actually higher during the visual only trials, suggesting that without any chemical information on female reproductive status the males continued to release urine, possibly in an attempt to stimulate the females because they did not appropriately respond across the sealed barrier. In contrast, when gravid females were in the same compartment as the subject male, and were detecting olfactory and other sensory information, urination rates were lower and courtship behavior rates were higher, presumably because they could more accurately determine the females' motivation and reproductive status, and the full interaction allowed for male reception of female 
chemical and behavioral responses. These results differ from those for the tilapia $O$. mossambicus, where males urinated more frequently in the full presence of receptive (pre-ovulatory) females than with unreceptive (post-ovulatory) females, but they did not change their courtship behavior rates based on female receptivity (Barata et al., 2008b). It is not known, however, whether the higher urination and lower courtship behavior rates observed in our $A$. burtoni visual only trials occurs in other fishes because previous studies have all introduced the stimulus female fish directly into the male's compartment (similar to the full interaction condition used here), and did not test the effects of unimodal sensory cues (Miranda et al., 2005; Barata et al., 2008b). While we ascribe the differences between the visual only and full interaction conditions in our experiments to additional sensory modalities, it is also possible that the visual information is qualitatively dissimilar between the two conditions because of differences in the proximity of the stimulus fish or their behavior. Further studies are needed to understand the valence of different visual signals in social contexts.

In a related study, however, Miranda and colleagues (Miranda et al., 2005) showed that anosmic (olfactory epithelium ablated) male tilapia $O$. mossambicus had reduced urination rates towards receptive females compared with males with intact olfactory epithelia, and suggested that a dominant male will only invest in chemical signaling if he can detect the female's reproductive readiness via olfactory cues. In contrast, our results suggest that visual cues alone are sufficient for dominant males to invest in chemical signaling, but not in courtship displays. Comparatively, it is likely more energetically demanding for a male to perform visual behavioral courtship displays than chemical signaling because their hyperosmotic physiology essentially provides a low-cost continuous vehicle for chemical release. Nevertheless, it is possible that dominant male $A$. burtoni release a compound in their urine that stimulates females to ovulate, and we speculate that without any olfactory feedback from the female to indicate whether she is receptive, the male may continue attempting to stimulate her by releasing more urine before investing energy in courtship behavior. The absence of a similar urination pattern towards mouth-brooding females suggests that dominant males can use visual cues alone to recognize that these females are not receptive and therefore do not invest in either chemical or behavioral signals. While the effects of male urine on female $A$. burtoni behavior and physiology require further study, chemical signals from males of other fish species are known to influence female behaviors, attraction towards males, spawning success and activity of the HPG axis (Sorensen, 1992; Fisher and Rosenthal, 2006; Gerlach, 2006; Barata et al., 2008a; Giaquinto et al., 2010; Stacey, 2011).

In addition to reproductive contexts, dominant male $A$. burtoni also use urine chemical signaling in the presence of other dominant males. This suggests that, in addition to visual signals such as coloration changes [e.g. eye-bar darkening during aggression (Heiligenberg et al., 1972)] and territorial behaviors [e.g. lateral displays, frontal threats, chases, bites, border fights (Fernald, 1977)], males may utilize chemical communication to convey social rank, territory ownership, motivation or aggression level. Thus, it is also possible that dominant male urine release serves to suppress nearby dominant males, regardless of the specific social context (e.g. territorial or reproductive). Dominant male tilapia O. mossambicus were also shown to store urine and then release it to actively advertise their dominance status, possibly to modulate aggression levels in rival males within the lek (Barata et al., 2007). Further, chemical communication decreases aggression levels in juvenile Nile tilapia $O$. niloticus possibly by increasing conspecific recognition and, therefore, stabilizing the dominance hierarchy (Giaquinto and Volpato, 1997). Similar to these studies in tilapia, when a dominant male A. burtoni was directly added to the subject males' compartment in our study, urination rates were lower and the subject male often stopped urinating once the two males had fought and the intruder faded his body and eye-bar coloration and became submissive, indicating that the resident subject had won (data not shown). The importance of chemosensory communication in these male-male interactions is further supported by the fact that urination rates were higher in the visual only trials where the signaler received no chemosensory information from the opponent. We know from another study in A. burtoni that visual stimuli from a larger dominant male can behaviorally suppress a smaller male, but that additional sensory cues are needed for full physiological suppression (Chen and Fernald, 2011). In A. burtoni, dominant males comprise a small percentage of the population, while the majority of males are subordinate, reproductively suppressed, school with females and do not hold territories (Fernald and Hirata, 1977; Fernald, 2009). These subordinate males are physically prevented from mating by aggression from dominant males, but our present results now suggest that urine release could possibly also serve as a chemical signal to reinforce or supplement the visual and physical social cues that suppress these subordinates within the population.

Evolution of pheromonal communication is predicted when signalers improve their reproductive success via changes in the way they produce or deliver a chemical cue, which can be driven by sexual selective pressures such as mate choice (Stacey and Sorensen, 2005; Swaney and Keverne, 2009). Our results showing that male A. burtoni alter their urine release in different social contexts now provide some support for the hypothesized pheromone communication system in this species. The importance of chemosensory signaling in A. burtoni is also supported by the finding that steroid receptor mRNA levels vary with reproductive state in females and social status in males, suggesting that internal physiological state may modulate olfactory perception of chemical signals according to reproductive condition (Maruska and Fernald, 2010b).

Electro-olfactogram (EOG) recordings previously demonstrated that $A$. burtoni responds to and can discriminate among various conjugated (glucuronidated and sulfated) steroids, but not unconjugated steroids or prostaglandins, which also suggests a complex pheromone system utilizing steroid conjugates (Robison et al., 1998; Cole and Stacey, 2006). Sulfated steroids are also used for chemical communication in mammals, suggesting a long evolutionary history for conjugated steroids as pheromones (Nodari et al., 2008). Simulated male territory intrusion in several other African cichlid species was also associated with changes in levels of conjugated urinary 11-KT components released into the water compared with relatively unchanged levels of free 11-KT fractions released via the gills, suggesting that social context affects male urine release (Hirschenhauser et al., 2008). While the chemical identity of compounds released in the urine is not yet known in $A$. burtoni, conjugated and unconjugated steroidal compounds have been measured from the urine of other fish species including cichlids (Oliveira et al., 1996; Rocha and Reis-Henriques, 1996; Frade et al., 2002; Barata et al., 2008b; Hirschenhauser et al., 2008; Stacey, 2011), and the concentration of urinary compounds and olfactory sensitivity (determined via EOG recordings) can depend on dominance and nutritional status (Oliveira et al., 1996; Frade et al., 2002; Barata et al., 2007; Barata et al., 2008b; Giaquinto et al., 2010).

In summary, we have shown for the first time that dominant male A. burtoni use urine pulses as contextual chemical signals for both 
intra-sexual (territorial) and inter-sexual (reproductive) interactions, and that urination frequency and rate of dominance behaviors depend on the type of sensory information available to the signaling male, providing support for the hypothesis that $A$. burtoni uses a chemical communication system similar to other freshwater fishes. Our results also highlight the significance of multimodal sensory information in potentially mediating both male-male and male-female chemical and visual signaling, and emphasize the importance of examining multiple signal channels used during specific behavioral contexts, like courtship, to fully understand the evolution of complex signaling mechanisms across vertebrates (Partan and Marler, 1999; Candolin, 2003; Narins et al., 2005). Future studies in A. burtoni will examine how this chemical information is used and integrated with other senses by the intended receivers to mediate appropriate behavioral decisions.

\section{ACKNOWLEDGEMENTS}

We thank Matthew Goodyear for help with data collection, Anoop Neboori for assistance with Fig. 1, and Scott Juntti, Caroline Hu, Russ Carpenter and the reviewers for insightful comments on an earlier version of the manuscript.

\section{FUNDING}

This research was funded by the Stanford University Undergraduate Research Fund, National Institutes of Health [NIH grant no. F32NS061431 to K.P.M.; NIH grant no. NS 034950 to R.D.F.] and National Science Foundation [grant no. IOS0923588 to R.D.F.]. Deposited in PMC for release after 12 months.

\section{REFERENCES}

Almeida, O. G., Miranda, A., Frade, P., Hubbard, P. C., Barata, E. N. and Canario, A. V. (2005). Urine as a social signal in the mozambique tilapia (Oreochromis mossambicus). Chem. Senses 30 Suppl. 1, i309-i310.

Appelt, C. W. and Sorensen, P. W. (1999). Freshwater fish release urinary pheromones in a pulsatile manner. In Advances in Chemical Signals in Vertebrates (ed. R. E. Johnston, D. Muller-Schwarze and P. W. Sorensen), pp. 247-256. New York: Kluwer.

Appelt, C. W. and Sorensen, P. W. (2007). Female goldfish signal spawning readiness by altering when and where they release a urinary pheromone. Anim Behav. 74, 1329-1338.

Barata, E. N., Hubbard, P. C., Almeida, O. G., Miranda, A. and Canario, A. V. (2007). Male urine signals social rank in the Mozambique tilapia (Oreochromis mossambicus). BMC Biol. 5, 54.

Barata, E. N., Serrano, R. M., Miranda, A., Noguiera, R., Hubbard, P. C. and Canario, A. V. (2008a). Putative pheromones from the anal glands of male blennies attract females and enhance male reproductive success. Anim. Behav. 75, 379-389.

Barata, E. N., Fine, J. M., Hubbard, P. C., Almeida, O. G., Frade, P., Sorensen, P. W. and Canario, A. V. (2008b). A sterol-like odorant in the urine of Mozambique tilapia males likely signals social dominance to females. J. Chem. Ecol. 34, 438-449.

Bradbury, J. W. and Vehrencamp, S. L. (1998). Principles of Animal Communication Sunderland, MA.: Sinauer Associates, Inc.

Candolin, U. (2003). The use of multiple cues in mate choice. Biol. Rev. Camb. Philos. Soc. 78, 575-595.

Castro, A. L., Goncalves-de-Freitas, E., Volpato, G. L. and Oliveira, C. (2009). Visual communication stimulates reproduction in Nile tilapia, Oreochromis niloticus (L.). Braz. J. Med. Biol. Res. 42, 368-374.

Chen, C. C. and Fernald, R. D. (2011). Visual information alone changes behavior and physiology during social interactions in a cichlid fish (Astatotilapia burtoni). PLOS One 6, e20313.

Cole, T. and Stacey, N. E. (2006). Olfactory responses to steroids in an African mouth-brooding cichlid, Haplochromis burtoni (Gunther). J. Fish Biol. 68, 661-680.

Coquelin, A. and Bronson, F. H. (1979). Release of luteinizing hormone in male mice during exposure to females: habituation of the response. Science 206, 1099-1101.

Crapon de Caprona, M. D. (1974). The effect of chemical stimuli from conspecifics on the behavior of Haplochromis burtoni (Cichlidae, Pisces). Experientia 30, 1394-1395. Crapon de Caprona, M. D. (1980). Olfactory communication in a cichlid fish, Haplochromis burtoni. Z. Tierpsychol. 52, 113-134.

Doving, K. (1976). Evolutionary trends in olfaction. In The Structure-Activity Relationships in Olfaction (ed. G. Benz), pp. 149-159. London, UK: IRL Press.

Dulac, C. and Torello, A. T. (2003). Molecular detection of pheromone signals in mammals: from genes to behaviour. Nat. Rev. Neurosci. 4, 551-562.

Dulka, J. L., Stacey, N. E., Sorensen, P. W. and Van der Kraak, G. J. (1987). A steroid sex pheromone synchronizes male-female spawning readiness in goldfish. Nature 325, 251-253.
Fernald, R. D. (1977). Quantitative behavioral observations of Haplochromis burtoni under semi-natural conditions. Anim. Behav. 25, 643-653.

Fernald, R. D. (2009). Social regulation of reproduction: what changes and why? Horm. Brain Behav. 1, 683-691.

Fernald, R. D. and Hirata, N. R. (1977). Field study of Haplochromis burtoni: quantitative behavioral observations. Anim. Behav. 25, 964-975.

Fisher, H. and Rosenthal, G. G. (2006). Female swordtail fish use chemical cues to select well-fed mates. Anim. Behav. 72, 721-725.

Frade, P., Hubbard, P. C., Barata, E. N. and Canario, A. V. (2002). Olfactory sensitivity of the Mosambique tilapia to conspecific odours. J. Fish Biol. 61, 12391254.

Gerlach, G. (2006). Pheromonal regulation of reproductive success in female zebrafish: female suppression and male enhancement. Anim. Behav. 72, 1119-1124.

Giaquinto, P. C. and Volpato, G. L. (1997). Chemical communication, aggression, and conspecific recognition in the fish Nile tilapia. Physiol. Behav. 62, 1333-1338.

Giaquinto, P. C., Militao da Silva Berbert, C. and Delicio, H. C. (2010). Female preferences based on male nutritional chemical traits. Behav. Ecol. Sociobiol. 64 1029-1035.

Heiligenberg, W., Kramer, U. and Schulz, V. (1972). The angular orientation of the black eye-bar in Haplochromis burtoni (Cichlidae, Pisces) and its relevance for aggressivity. Z. Vergl. Physiol. 76, 168-176.

Hirschenhauser, K., Taborsky, M., Oliveira, T., Canario, A. V. and Oliveira, R. F. (2004). A test of the 'challenge hypothesis' in cichlid fish: simulated partner and territory intruder experiments. Anim. Behav. 68, 741-750.

Hirschenhauser, K., Canario, A. V., Ros, A. F., Taborsky, M. and Oliveira, R. F. (2008). Social context may affect urinary excretion of 11-ketotestosterone in African cichlids. Behavior 145, 1367-1388.

Johnston, R. E. and Bronson, F. (1982). Endocrine control of female mouse odors that elicit luteinizing hormone surges and attraction in males. Biol. Reprod. 27, 11741180.

Karlson, P. and Luscher, M. (1959). Pheromones: a new term for a class of biologically active substances. Nature $385,725-729$.

Kodric-Brown, A. and Strecker, U. (2001). Responses of Cyprinodon maya and $C$. labiosus females to visual and olfactory cues of conspecific and heterospecific males. Biol. J. Linnean Soc. 74, 541-548.

Maruska, K. P. and Fernald, R. D. (2010a). Steroid receptor expression in the fish inner ear varies with sex, social status, and reproductive state. BMC Neurosci. 11 58.

Maruska, K. P. and Fernald, R. D. (2010b). Reproductive status regulates expression of sex steroid and $\mathrm{GnRH}$ receptors in the olfactory bulb. Behav. Brain. Res. 213, 208-217.

Miranda, A., Almeida, O. G., Hubbard, P. C., Barata, E. N. and Canario, A. V. (2005). Olfactory discrimination of female reproductive status by male tilapia (Oreochromis mossambicus). J. Exp. Biol. 208, 2037-2043.

Narins, P. M., Grabul, D. S., Soma, K. K., Gaucher, P. and Hodl, W. (2005). Crossmodal integration in a dart-poison frog. Proc. Natl. Acad. Sci. USA 102, 2425 2429.

Nodari, F., Hsu, F. F., Fu, X., Holekamp, T. F., Kao, L. F., Turk, J. and Holy, T. E. (2008). Sulfated steroids as natural ligands of mouse pheromone-sensing neurons. J. Neurosci. 28, 6407-6418.

Oliveira, R. F., Almada, V. and Canario, A. V. (1996). Social modulation of sex steroid concentrations in the urine of male cichlid fish Oreochromis mossambicus. Horm. Behav. 30, 2-12.

Partan, S. R. and Marler, P. (1999). Communication goes multimodal. Science $\mathbf{2 8 3}$ $1272-1273$.

Robison, R. R., Fernald, R. D. and Stacey, N. E. (1998). The olfactory system of a cichlid fish responds to steroidal compounds. J. Fish Biol. 53, 226-229.

Rocha, M. J. and Reis-Henriques, M. A. (1996). Plasma and urine levels of $C_{18}, C_{19}$ and $\mathrm{C}_{21}$ steroids in an asynchronous fish, the tilapia Oreochromis mossambicus (Teleostei, Cichlidae). Comp. Biochem. Physiol. 115C, 257-264.

Rosenthal, G. G., Fitzsimmons, J. N., Woods, K. U., Gerlach, G. and Fisher, H. S (2011). Tactical release of a sexually-selected pheromone in a swordtail fish. PLoS ONE 6, e16994.

Sorensen, P. (1992). Hormonally derived sex pheromones in goldfish: a model for understanding the evolution sex pheromone systems in fish. Biol. Bull. 183, 173-177.

Sorensen, P. W., Scott, A. P. and Kihslinger, R. L. (2000). How common hormonal metabolites function as relatively specific pheromonal signals in the goldfish. In Proceedings of the 6th International Symposium on Reproductive Physiology of Fish (ed. B. Norberg, O. S. Kjesbu, G. L. Taranger, E. Andersson and S. O. Stefansson), pp. 125-128. Bergen, Norway.

Stacey, N. (2011). Hormonally derived sex pheromones in fishes. In Hormones and Reproduction of Vertebrates, Vol. 1, Fishes (ed. D. O. Norris and K. H. Lopez), pp. 169-192. Oxford, UK: Elsevier.

Stacey, N. and Sorensen, P. W. (2005). Reproductive pheromones. In Behavior and Physiology of Fish (ed. S. Sloman, R. W. Wilson and S. Balshine), pp. 359-412. London: Academic Press.

Swaney, W. T. and Keverne, E. B. (2009). The evolution of pheromonal communication. Behav. Brain. Res. 200, 239-247.

Van Staaden, M. J. and Smith, A. R. (2011). Cutting the Gordian knot: complex signaling in African cichlids is more than multimodal. Curr. Zool. 57, 237-252. 